\title{
Null Extinction of Ceria@silica Hybrid Particles: Transparent Polystyrene Composites
}

\author{
Anıl İncel, Tuğrul Güner, Onur Parlak, ${ }^{\dagger}$ and Mustafa M. Demir* \\ Department of Materials Science and Engineering, İzmir Institute of Technology, Gülbahçe Köyü, Urla, 35430 İzmir, Turkey
}

Supporting Information

ABSTRACT: Scattering of light in optical materials, particularly in composites based on transparent polymer and inorganic pigment nanoparticles, is a chronic problem. It might originate mainly from light scattering because of a refractive index mismatch between the particles and transparent polymer matrix. Thus, the intensity of light is rapidly diminished and optical transparency is reduced. Refractive index matching between the pigment core and the surrounding transparent matrix using a secondary component at the interface (shell) has recently appeared as a promising approach

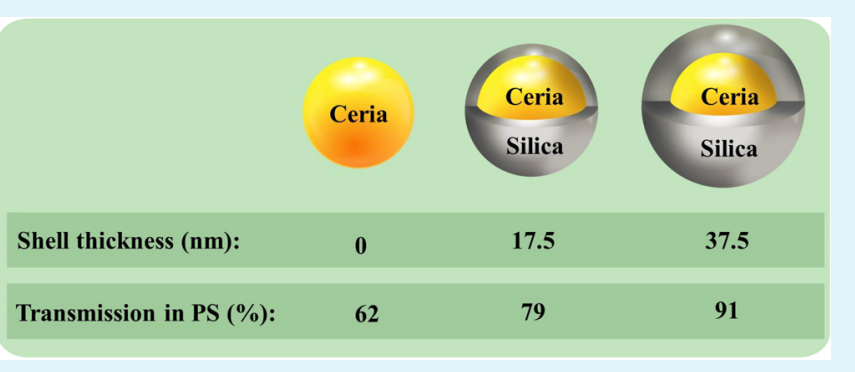
to alter light scattering. Here, $\mathrm{CeO}_{2}$ (ceria) nanoparticles with a diameter of $25 \mathrm{~nm}$ are coated with a $\mathrm{SiO}_{2}$ (silica) shell with various thicknesses in a range of 6.5-67.5 nm using the Stöber method. When the hybrid core-shell particles are dispersed into transparent polystyrene (PS), the transmission of the freestanding PS composite films increases over both the ultraviolet (UV) and visible region as the shell thickness increases particularly at $37.5 \mathrm{~nm}$. The increase of transmission can be attributed to the reduction in the scattering coefficient of the hybrid particles. On the other hand, the particles in tetrahydrofuran (THF) absorb over UV and the intensity of absorption shows a systematic decrease as the shell thickness increases. Thus, the silica shell suppresses not only the scattering coefficient but also the molar absorptivity of the core ceria particles. The experimental results regarding the target shell thickness to develop low extinction (scattering + absorption) composites show a qualitative agreement with the predictions of Effective Medium Theory.

KEYWORDS: absorption, core-shell particles, index matching, polymer nanocomposites, light scattering

\section{INTRODUCTION}

Nanosized crystalline particles are critically important to designing novel optical composite materials where particulate pigments are added to a transparent matrix to develop optical features like absorption or emission at a desired wavelength of the optical spectrum, ${ }^{1,2}$ wave guiding the electromagnetic radiation, ${ }^{3}$ optical limiters, ${ }^{4}$ or high/low refractive indices to control the focusing power of the materials. ${ }^{5}$ However, there is often a trade-off between the development of one or more novel optical features and the optical transparency. ${ }^{6}$ Because of the sharp refractive index (RI) at the interface of particles and polymers, transparency suffers from strong light scattering and absorption, and eventually transparency is deteriorated. ${ }^{5-13}$ The reduction of size of the scatterers (individual particles and particle domains) has been the most common approach for suppressing optical scattering. ${ }^{3,14,15}$ When the size of scatterers is smaller than $1 / 20$ of the wavelength of electromagnetic radiation, the radiation propagates throughout a material without scattering according to Rayleigh scattering. ${ }^{16-18}$ The size of the particle domains should be in the range of 20-40 $\mathrm{nm}$ to maintain the transparency in polymeric matrices. However, it is hard to keep these nanometer-sized particles individual against the aggregation. ${ }^{19}$ Upon aggregation, the size of particle domains becomes larger and strong scattering is unavoidable. ${ }^{3,20}$ There are many elegant discussions, reviews have been published, and material scientists have continuously tried to overcome these technical hurdles with optical nanocomposites. 5,12

When refractive index of guest particles is close to the surrounding polymer matrix, the difference in $\mathrm{RI}$ is reduced and the transparency of the matrix is maintained. ${ }^{21,22}$ Therefore, the choice of material components plays a key role in optical composites. For instance, silica particles have been commonly used with poly(methyl methacrylate) (PMMA) where their indices are close to each other such that $n_{\mathrm{PMMA}}=1.49$ and $n_{\text {silica }}$ $=1.46$ at $633 \mathrm{~nm} .^{22}$ On the other hand, the increased RI of a polymer matrix can be another approach to reduce the RI mismatch between particle and matrix. Polymers traditionally have relatively low refractive indices, but they can be raised to technologically useful values using brominated and aromatic monomers to avoid the scattering of high RI materials. ${ }^{21}$ However, the number of potential polymers and pigments whose RI is close to each other is scarce.

Refractive index matching between a guest inorganic pigment particle and the surrounding host polymer matrix has recently been demonstrated as an efficient way to suppress scattering. ${ }^{23}$

Received: October 15, 2015

Accepted: November 23, 2015

Published: November 23, 2015 
Core-shell particle morphology has attracted particular interest where a high refractive index core material is coated with a lower index of shell such that the effective refractive index is substantially equal to the refractive index of the embedding matrix medium. Thus, incident light sees one uniform particle with an efficient refractive index since there is no sharp refractive index increase at the interface. The key point in the design of the core-shell particles for the aim of index matching is the homogeneous coating of the shell material with a target thickness and composition. Previously, Bockstaller and Matyjaszewski demonstrated the minimization of scattering for polystyrene-grafted $\mathrm{SiO}_{2}$ nanoparticles in liquid state (toluene). ${ }^{6}$ The precise control of the $\mathrm{SiO}_{2}$ layer thickness is essential for the design. Optimum polymer-graft composition for the shell is obtained using atom transfer radical polymerization (ATRP) for a well-defined coating on the $\mathrm{SiO}_{2}$ core with a controlled graft density, molecular weight, and appropriate composition. ${ }^{24}$ Our group previously developed a method to increase the transparency of $\mathrm{CeO}_{2}$ particles grafted with PMMA in a PS matrix. ${ }^{25}$ PMMA is coated to the surface of ceria nanoparticles via free radical polymerization, which allowed us to control the thickness of the PMMA shell by tuning the initiator concentration. There is an inverse relation between the shell thickness and the amount of initiator employed in the solution polymerization process. It should be noted that the incompatibility between the PMMA shell and the PS matrix chains causes phase separation and is indeed an additional source for optical scattering in the system because of the refractive index mismatch between the polymers. ${ }^{26}$ The selection of an amorphous inorganic material as a shell component may help overcome this incompatibility and improve the transparency. In this context, silica can be considered a good candidate for a shell to obtain transparent optical composites because of its ease of deposition, chemical inertness, low refractive index, and glassy nature. ${ }^{27}$ To the best of our knowledge, $\mathrm{Li}$ et al. was the first to report experimental validation of index matching phenomenon. ${ }^{28}$ The authors showed that $\mathrm{SiO}_{2}$ coating on $\mathrm{TiO}_{2}$ particles using the Stöber method enabled the core-shell particles to act as integral filler in an epoxy matrix. The scattering is strongly suppressed and high transparency is achieved for thick composite film when the weight percentage of silica shell is $35 \%$ by mass for $1 \mathrm{wt} \% \mathrm{TiO}_{2}$ pigment core. However, the effect of silica shell on the optical absorption of the $\mathrm{TiO}_{2}$ particles was not investigated.

The aim of the current study is to examine the extinction feature of the core-shell type ceria-silica particles and their composites with a model PS matrix over a wide range of wavelengths including the UV-visible region. Absorption and scattering are two extinction processes upon which the intensity of the incident beam is gradually attenuated. The extinction determines how far an incident light of a particular wavelength can penetrate into before it is scattered or absorbed. The control over the magnitude of the extinction coefficient can play a drastic role for various photosystems like the collector efficiency of a photo thermal energy conversion ${ }^{29}$ or a line width in a lithograph process. ${ }^{30}$ Here, the composition of the ceria core and silica shell governs this extinction feature. Effective Medium Theory has been used for the design of core-shell morphology.

\section{EXPERIMENTAL DESIGN}

Materials and Methods. The dispersion of cerium(IV) oxide nanoparticles $\left(\mathrm{CeO}_{2} \mathrm{NPs}\right)$ in $\mathrm{H}_{2} \mathrm{O}\left(\leq 25 \mathrm{~nm} 10\right.$ wt $\%$ in $\left.\mathrm{H}_{2} \mathrm{O}\right)$, tetraethyl orthosilicate (99.99\% trace metals basis), ammonia solution (anhydrous, $\geq 99.9 \%$ ), tetrahydrofurane (THF, 99.9\%), ethanol (EtOH) ( $\geq 99.8 \%)$, polyvinylpyrrolidone (PVP) $(\sim 8 \mathrm{kDa})$, and polystyrene $(\sim 350 \mathrm{kDa})$ were purchased from Sigma-Aldrich (St. Louis, MO, USA). All other reagents and solvents are at analytical grade and were purified prior to use. A Malvern dynamic light scattering (DLS) Nano-ZS instrument (Worcestershire, UK) was used for size and zeta potential measurements. UV-vis spectra were recorded on a Varian Cary $50 \mathrm{UV}$-vis spectrophotometer (Palo Alto, USA). Scanning electron microscopy, FEI Quanta 250 Feg (Oregon, USA) was used in the mode of backscattered at high voltage to acquire images. An X-ray diffractometer, the Philips X'Pert Pro (Eindhoven, Netherlands), was used for elemental analysis.

Synthesis of $\mathrm{Neat}^{\mathrm{CeO}_{2}}$ Nanoparticles. A sample of $1.0 \mathrm{~g}$ PVP was dissolved into $20 \mathrm{~mL}$ of distilled $\mathrm{H}_{2} \mathrm{O}$ and the solution was sonicated for $30 \mathrm{~min}$. An aliquot of $3 \mathrm{~mL}$ of $\mathrm{CeO}_{2}$ dispersion was diluted by $50 \mathrm{~mL} \mathrm{H}_{2} \mathrm{O}$. The solution and dispersion were added into the same container for further mixing. The solution was kept at 400 rpm for $24 \mathrm{~h}$ to modify the surface of $\mathrm{CeO}_{2}$ with PVP. After this modification process, the solution was centrifuged and washed three times successively with distilled water to remove unbound polymer chains. The precipitate was dried under vacuum at $100{ }^{\circ} \mathrm{C}$ for $2 \mathrm{~h}$.

Synthesis of $\mathrm{CeO}_{2} @ \mathrm{SiO}_{2}$ Core-Shell Nanoparticles. To obtain successful incorporation of $\mathrm{SiO}_{2}$ into the PVP-coated $\mathrm{CeO}_{2}$ particles, we set $\mathrm{pH}$ of the medium to 9 . The solvent mixture was prepared from $\mathrm{NH}_{3}: \mathrm{EtOH}: \mathrm{H}_{2} \mathrm{O}$ solution and the volume fractions were determined as 0.4:1.0:2.0, respectively. This solution was mixed for $1 \mathrm{~h}$ to regulate the $\mathrm{pH}$ condition. On the other hand, $60 \mathrm{mg}$ from $\mathrm{CeO}_{2} / \mathrm{PVP}$ dried powder was dispersed in $5 \mathrm{~mL} \mathrm{H}_{2} \mathrm{O}$ by ultrasonication. When the dispersion process was complete, this solution was injected into the solvent mixture dropwise. After $30 \mathrm{~min}$, the solution was evenly separated into four different containers. At the same time, various concentrations of TEOS [1.08 M, 0.54 M, $0.108 \mathrm{M}, 0.054 \mathrm{M}$ ] were prepared by dissolving required amount of TEOS in $5 \mathrm{~mL}$ of EtOH. Note that the brackets refer to concentration; for instance, [TEOS] refers to the concentration of TEOS. Each $\mathrm{CeO}_{2} / \mathrm{PVP}$ solution was treated with ethanol-TEOS solution, successively. The $\mathrm{SiO}_{2}$ coating onto the $\mathrm{CeO}_{2}$ core took place $12 \mathrm{~h}$ under continuous stirring. After the reactions were terminated, each solution was centrifuged and the precipitate was vacuum-dried at $100{ }^{\circ} \mathrm{C}$ for $2 \mathrm{~h}$.

Preparation of PS-CeO $\mathrm{CSiO}_{2}$ Nanocomposite Films. A 15\% PS solution by mass was prepared in THF. For each film preparation, core-shell nanoparticles were taken into $1 \mathrm{~mL}$ of PS polymer solution. After sonication of the dispersions, composite films were obtained using a model WS 400B Spin Coater (Laurell Technologies Corp., North Wales). Then, the films were annealed at $100{ }^{\circ} \mathrm{C}$ for $2 \mathrm{~h}$. The amount of core with respect to PS matrix was fixed at $3 \%$ by mass. Size distribution of particle domains was measured using a freeware software named ImageJ. ${ }^{31}$

Theoretical Background for Optical Properties of the CoreShell Particles. The scattering and extinction coefficients are designated $C_{\text {sca }}$ and $C_{\text {ext }}$, respectively. These coefficients were calculated using Mie theory and following the Bohren and Huffman methods. ${ }^{41}$ The refractive index of the core $n_{\mathrm{CeO} 2}=2.20$, shell $n_{\mathrm{SiO} 2}=$ 1.46 , and surrounding matrix $n_{\mathrm{PS}}=1.59$ are taken at $633 \mathrm{~nm}{ }^{32}$ The calculation was based on the Fortran program given in the book by Bohren and Huffmann, and this code was adjusted to Matlab ${ }^{33}$ (Supporting Information). Typically, the calculation of scattering was performed as follows

$$
\begin{aligned}
& C_{\mathrm{Sca}}=\frac{2}{y^{2}} \sum_{n=1}^{\infty}(2 n+1)\left(\left|a_{n}\right|^{2}+\left|b_{n}\right|^{2}\right) \\
& C_{\mathrm{Ext}}=\frac{2}{y^{2}} \sum_{n=1}^{\infty}(2 n+1)\left(\operatorname{Re}\left(a_{n}\right)+\operatorname{Re}\left(b_{n}\right)\right)
\end{aligned}
$$




$$
\begin{aligned}
& a_{n}=\frac{\psi_{n}(y)\left[\widetilde{\left[D_{n}\right.} / m_{2}+n / y\right]-\psi_{n-1}(y)}{\xi_{n}(y)\left[\widetilde{D}_{n} / m_{2}+n / y\right]-\xi_{n-1}(y)} \\
& b_{n}=\frac{\psi_{n}(y)\left[m_{2} \widetilde{G}_{n}+n / y\right]-\psi_{n-1}(y)}{\xi_{n}(y)\left[m_{2} \widetilde{G}_{n}+n / y\right]-\xi_{n-1}(y)}
\end{aligned}
$$

and with

$$
\begin{aligned}
& \widetilde{D}_{n}=\frac{D_{n}\left(m_{2} y\right)-A_{n} \chi_{n}^{\prime}\left(m_{2} y\right) / \psi_{n}\left(m_{2} y\right)}{1-A_{n} \chi_{n}\left(m_{2} y\right) / \psi_{n}\left(m_{2} y\right)} \\
& \widetilde{G}_{n}=\frac{D_{n}\left(m_{2} y\right)-B_{n} \chi_{n}^{\prime}\left(m_{2} y\right) / \psi_{n}\left(m_{2} y\right)}{1-B_{n} \chi_{n}\left(m_{2} y\right) / \psi_{n}\left(m_{2} y\right)} \\
& A_{n}=\psi_{n}\left(m_{2} x\right) \frac{\left(\frac{m_{2}}{m_{1}}\right) D_{n}\left(m_{1} x\right)-D_{n}\left(m_{2} x\right)}{\left(\frac{m_{2}}{m_{1}}\right) D_{n}\left(m_{1} x\right) \chi_{n}\left(m_{2} x\right)-\chi_{n}^{\prime}\left(m_{2} x\right)} \\
& B_{n}=\psi_{n}\left(m_{2} x\right) \frac{\left(\frac{m_{2}}{m_{1}}\right) D_{n}\left(m_{2} x\right)-D_{n}\left(m_{1} x\right)}{\left(\frac{m_{2}}{m_{1}}\right) \chi_{n}^{\prime}\left(m_{2} x\right)-D_{n}\left(m_{1} x\right) \chi_{n}\left(m_{2} x\right)}
\end{aligned}
$$

In general, the coefficients $a_{n}$ and $b_{n}$ are known as Mie scattering coefficients; $m_{1}=n_{\text {core }} / n_{\text {medium }}$ and $m_{2}=n_{\text {shell }} / n_{\text {medium }}$ are the relative refraction indices of both the core and shell to the medium; and $x=$ $k R_{0}$ and $y=k R_{\mathrm{p}}$ are the size parameters, where $k=2 \pi n_{\text {medium }} / \lambda(\lambda=$ $550 \mathrm{~nm})$, and $R_{0}(=12.5 \mathrm{~nm})$ and $R_{\mathrm{p}}$ represent the core radius and core-shell particle radius, respectively. $D_{n}$ is the logarithmic derivative of $\psi\left(D_{n}=\psi^{\prime}{ }_{n} / \psi_{n}\right) ; \psi_{n}(z)$ and $\chi_{n}(z)$ are Ricatti-Bessel functions; and $\xi_{n}(z)$ is the Hankel function $\left(\psi_{n}(z)=z j_{n}(z), \chi_{n}(z)=z y_{n}(z)\right.$ and $\xi_{n}(z)$ $=z\left(j_{n}(z)+i y_{n}(z)\right)$, where $j_{n}(z)$ and $y_{n}(z)$ represent the spherical Bessel functions).

\section{RESULTS AND DISCUSSION}

Scheme 1 outlines the entire process for the preparation of PS/ $\mathrm{CeO}_{2} @ \mathrm{SiO}_{2}$ composites. The synthesis strategy is, first, to form

Scheme 1. Schematic Diagrams for the Preparation of Optical PS Composite Films Prepared by Silica-Coated $\mathrm{CeO}_{2}$ Hybrid Particles

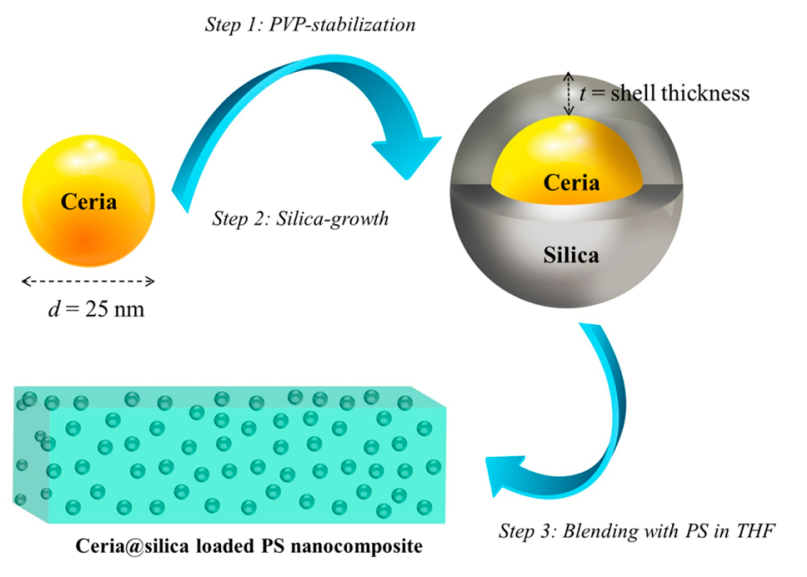

core-shell type nanostructured hybrid particles with a controlled shell thickness. This is the key step for controlling the transparency of the composite material. The next step involves solution blending these particles with a transparent polymer. The polymer/particle dispersion in THF is then cast on a substrate to obtain an optical composite film.
Ceria Core-Silica Shell Particles. A commercial suspension consisting of $25 \mathrm{~nm}$ of ceria nanoparticles was employed as a particle core. It is common to prepare a stable colloid prior to the silica coating process. PVP was used to stabilize ceria colloids in THF in this work. The particles were sterically stabilized by PVP to prevent undesirable aggregation of the core particles. PVP is employed as a coupling agent, which is an amphiphilic, nonionic polymer that can easily adsorb on oxidic surfaces. ${ }^{34}$ Moreover, PVP makes the affinity of the ceria surface to silica sufficiently high via hydrogen bonding between amide carbonyl of PVP and silanol, which helps to achieve uniform silica coating over ceria core. ${ }^{35}$ It provides stabilization of $\mathrm{CeO}_{2}$ particles in a water/ethanol mixture by adsorbing onto the surface of particles. Stöber is a common method used for the formation of silica wherein the reaction mechanism is based on hydrolysis and condensation of TEOS. ${ }^{36}$ Fine control of [TEOS] allows for the preparation of ceria-silica core-shell particles with controllable shell thicknesses. Figure 1 illustrates the X-ray diffractogram of the $\mathrm{CeO}_{2}$

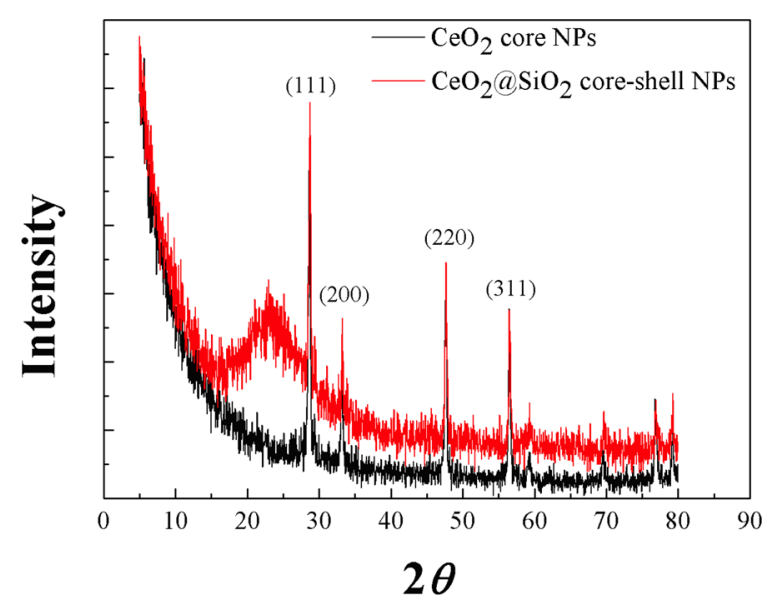

Figure 1. X-ray diffraction pattern for $\mathrm{CeO}_{2}$ core and $\mathrm{CeO}_{2} @ \mathrm{SiO}_{2}$ core-shell nanoparticles.

core and $\mathrm{CeO}_{2} @ \mathrm{SiO}_{2}$ core-shell particles. The diffractogram of ceria core exhibits sharp reflections that are perfectly indexed with the signals of ceria (JCPDS: 01-075-0390). When the core is covered with silica shell, along with the characteristic reflections of ceria, a broad signal centered at $24^{\circ}$ appears. This signal is known as an "amorphous halo" and is an indication of the presence of silica in the system.

Figure $2 \mathrm{x}$ shows the number size distribution of PVPmodified $\mathrm{CeO}_{2}$ (core) and $\mathrm{CeO}_{2} @ \mathrm{SiO}_{2}$ core-shell particles prepared in the presence of different amounts of TEOS. The PVP-capped $\mathrm{CeO}_{2}$ core exhibits narrow size distribution with a mean diameter of $25 \mathrm{~nm}$. Upon coating the ceria core with silica shell, the diameter of the hybrid particles shifts to a larger mean diameter (as shown in column two of Table 1) and the size distributions become broader. The precipitation of $\mathrm{SiO}_{2}$ most probably occurs on the surface of ceria following heterogeneous nucleation via sol-gel reaction. The formation of free silica particles without a ceria core, i.e., homogeneous nucleation, and the nucleation of hybrid particles with multiple ceria cores is avoided. Note that heterogeneous nucleation on a surface requires lower energy so that nucleation on the surface is more favorable. ${ }^{37}$

One can roughly estimate the thickness of the silica shell by simply subtracting the diameter of the core $(25 \mathrm{~nm})$ from the 

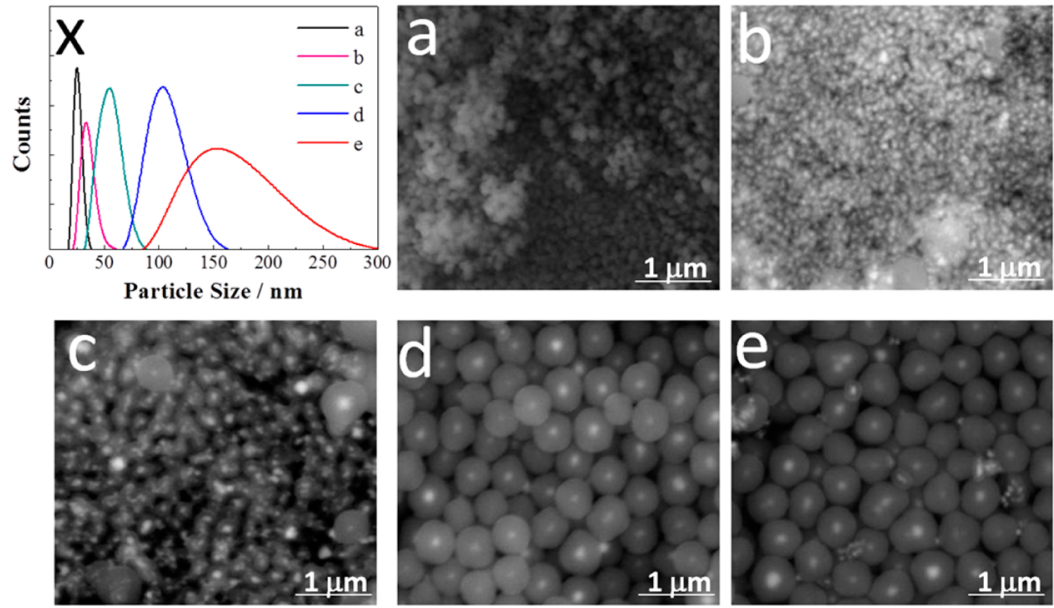

Figure 2. (x) Number size distributions of $\mathrm{CeO}_{2} / \mathrm{PVP}$ (neat) and $\mathrm{CeO}_{2} @ \mathrm{SiO}_{2}$ core-shell particles prepared at different [TEOS] in the course of particle synthesis. SEM images of $(a)$ neat $\mathrm{CeO}_{2}$ and $(\mathrm{b}-\mathrm{e}) \mathrm{CeO}_{2} @ \mathrm{SiO}_{2}$ nanoparticles with increasing shell thickness from left to right of (b) 6.5, (c) 17.5 , (d) 37.5 , and (e) $67.5 \mathrm{~nm}$. The letters have been used consistently for the samples.

Table 1. Mean Diameter and Dielectric Constant of CoreShell Hybrid Particles $\left(\varepsilon_{\text {eff }}\right)^{a}$

$\begin{array}{lccccc}\text { [TEOS }] & R_{\text {core-shell }}(\mathrm{nm}) & \mathrm{SiO}_{2} \text { shell }(\mathrm{nm}) & \varepsilon_{\text {eff }} & n & \% T \\ 0 & 25 & 0 & 4.84 & 2.2 & 62 \\ 0.054 & 38 & 6.5 & 7.75 & 1.8 & 76 \\ 0.011 & 60 & 17.5 & 6.12 & 1.67 & 79 \\ 0.540 & 100 & 37.5 & 5.14 & 1.59 & 91 \\ 1.080 & 160 & 67.5 & 4.64 & 1.54 & 91\end{array}$

${ }^{a}$ Index matching condition is highlighted with bold.

mean diameter of core-shell system. The result of this estimation is given in column three of Table 1 for the corresponding [TEOS]. The thickness of the silica shell can be readily controlled by [TEOS]. For example, the mean diameter of the hybrid particles was $100 \mathrm{~nm}$ when the amount of [TEOS] was $0.540 \mathrm{M}$. As [TEOS] was reduced 10-fold to $0.054 \mathrm{M}$, the mean particle size distribution decreased gradually to $38 \mathrm{~nm}$. As a result, the thickness of $\mathrm{SiO}_{2}$ shell is readily controlled by the concentration of TEOS in the course of the particle synthesis considering that the diameter of core is fixed. Figure 2a-e show scanning electron microscopy (SEM) images of PVP-stabilized $\mathrm{CeO}_{2}$ (core) and $\mathrm{CeO}_{2} @ \mathrm{SiO}_{2}$ core-shell particles with different thickness. The images were obtained from the powder of the particles. The neat $\mathrm{CeO}_{2}$ particles are in aggregated structure. Backscattered electron imaging mode at $15 \mathrm{kV}$ was applied to determine the color contrast between the massive core and the lighter silica shell. The core and the shell hybrid particles are concentric spheres and the core appears to be bright since the electron density of ceria is higher. The core seems to be homogeneously covered with silica shell. As the [TEOS] increases in the particle synthesis, the thickness of the $\mathrm{SiO}_{2}$ shell obviously increases (Figure $2 \mathrm{~b}-\mathrm{e}$ ). The results obtained by microscopy are compatible with the size of coreshell particles measured from DLS.

Figure 3a shows the UV-vis absorption spectra of ceria core and ceria@silica hybrid particles in aqueous medium. The spectrum of neat $\mathrm{CeO}_{2}$ shows an intense absorption signal at $315 \mathrm{~nm}$, which is attributed to the absorption band of ceria. Not surprisingly, it is a semiconductor and absorbs the UVregion of the optical spectrum. The band gap is calculated at $3.26 \mathrm{eV}$. For the $\mathrm{CeO}_{2} @ \mathrm{SiO}_{2}$ systems, the absorption signal is remarkably reduced when the ceria core is surrounded by silica
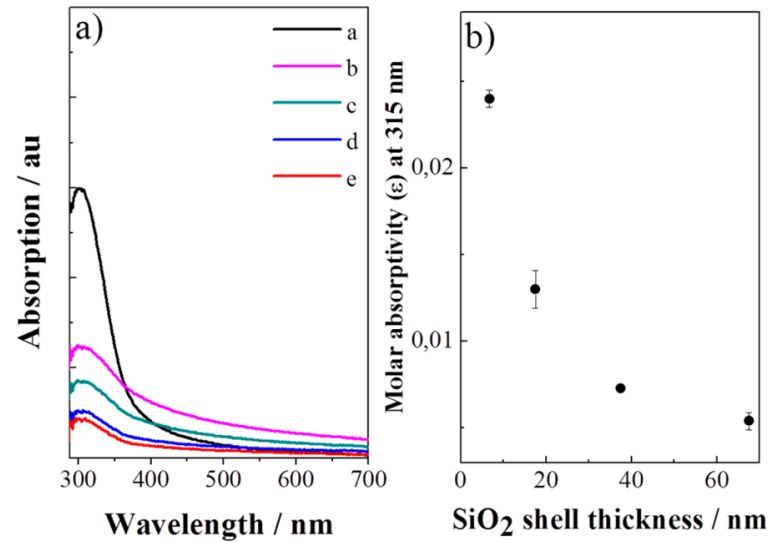

Figure 3. (a) Absorption spectrum of neat and silica-coated $\mathrm{CeO}_{2}$ nanoparticles prepared at different [TEOS]. The solutions are dispersed in THF solution at $\mathrm{pH} 9$ using equal amount of particles. The spectra of the hybrid particles: (a) neat $\mathrm{CeO}_{2}$ and (b-e) $\mathrm{CeO} 2 @$ $\mathrm{SiO}_{2}$ nanoparticles with increasing shell thickness from top to bottom of (b) 6.5, (c) 17.5, (d) 37.5, and (e) $67.5 \mathrm{~nm}$. (b) Molar absorptivity of the hybrid particles in THF taking Beer's law into account.

shell. Moreover, the signal is systematically diminished as the shell becomes thicker. Previously, it was shown that silica coating remarkably affects the optical feature of metal nanoparticles. For instance, the signal of surface plasmon resonance of $\mathrm{Au}^{38}$ and $\mathrm{Ag}^{39}$ is affected and exhibited red shift by silica shell thickness. Moreover, the coating of chalcogenide particles with silica causes the broadening of the emission signal with reduced intensity. ${ }^{40}$ In this reference, it is shown that the quantum yield of CdSe particles is enhanced by silica shell coating. However, the reduction of the extinction coefficient is reported for the first time in our work according to the best of our knowledge.

Molar absorptivity of the hybrid particles was evaluated from the absorption spectra using Beer's law: $A=\varepsilon b c$, where $A$ is absorption, $\varepsilon$ is molar absorptivity, $b$ is path length, and $c$ is concentration of the particles. Figure $3 b$ presents the molar absorptivity of the particles as a function of shell thickness. It shows an exponential decay as the shell thickness increases.

Core-Shell Particles in Polystyrene. The effective dielectric constant $\left(\varepsilon_{\text {eff }}\right)$ and corresponding refractive index of 
core-shell hybrid particles can be estimated by the following Maxwell-Garnett equation. ${ }^{41}$ The result is presented in column four of Table 1 .

$$
\varepsilon_{\text {eff }}=\varepsilon_{\text {shell }}\left(1+3 \frac{\phi x}{1-\phi x}\right)
$$

where $x=1 / 3\left(\varepsilon_{\text {core }}-\varepsilon_{\text {shell }}\right) /\left(\varepsilon_{\text {core }}-1 / 3\left(\varepsilon_{\text {core }}-\varepsilon_{\text {shell }}\right)\right)$, where $\varepsilon_{\text {core }}$ and $\varepsilon_{\text {shell }}$ are the dielectric constant of core and shell of the particles, respectively, and $\phi=V_{\text {core }} /\left(V_{\text {core }}+V_{\text {shell }}\right)$ is the relative core volume. Note that the dielectric constant is related to the refractive index as $\varepsilon=n^{2}{ }^{42}$ Column five of Table 1 presents the estimated refractive index of the hybrid particles. Figure 4 illustrates the calculated refractive index of the hybrid

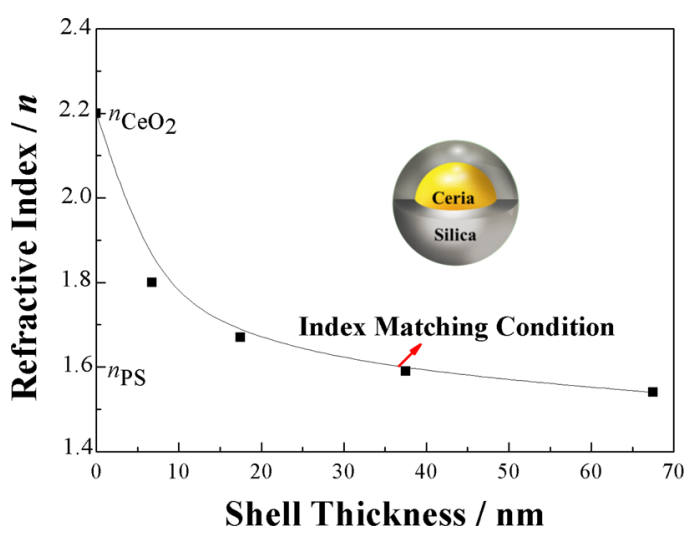

Figure 4. Effective refractive index of $\mathrm{SiO}_{2}$-coated $\mathrm{CeO}_{2}$ core-shell particles as a function of shell thickness calculated using MaxwellGarnett equation and assuming $n_{\mathrm{CeO} 2}=2.20$ and $n_{\mathrm{SiO} 2}=1.46$ at 633 $\mathrm{nm}$. The dashed line indicated the refractive index of PS $\left(n_{\mathrm{PS}}=1.59\right)$. Red line illustrated predicted index-matching condition in PS medium.

particles based on the Maxwell-Garnett equation. Neat $\mathrm{CeO}_{2}$ has a refractive index of 2.2 at $633 \mathrm{~nm},{ }^{25}$ and the one of PS is $1.59 .^{43}$ When silica is coated on the surface, the calculation shows an exponential decrease. Refractive index-matching of the core-shell in PS is satisfied at $37.5 \mathrm{~nm}$ shell thickness. With a further increase in the shell thickness for a core diameter of 25 $\mathrm{nm}$, the refractive index of the hypothetical hybrid particles asymptotically approaches that of neat silica.

The hybrid particles were dispersed into transparent atactic PS, which is a polymer matrix with a relatively higher refractive index than other transparent commodity polymers such as PMMA. Its refractive index lies between the high index ceria core and low index shell materials, as shown in Figure 4 and eq 9.

Figure 5 illustrates the scanning electron microscopy image of the silica-coated ceria particles embedded in the PS matrix. Even though the PS matrix is filled with core-shell particles around $5 \%$ by mass depending on thickness, the particles are not clearly visible beneath the PS due to the strong shielding of the matrix. However, some parts of the image show that the particles are well dispersed inside the matrix and that they kept their individual sizes without aggregation. This shows the positive effect of surface coating with silica on the ceria core, which helps to disperse the hybrid particles throughout the matrix volume.

Figure 6 shows the UV-vis transmission spectra of pristine PS and PS composites prepared by neat $\mathrm{CeO}_{2}$ and $\mathrm{CeO}_{2} @ \mathrm{SiO}_{2}$ hybrid particle systems with various thicknesses. The spectra

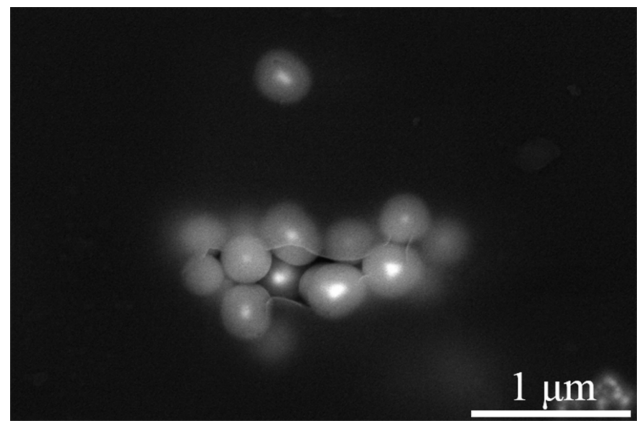

Figure 5. Representative SEM image of $\mathrm{PS} / \mathrm{CeO}_{2} @ \mathrm{SiO}_{2}$ nanocomposite film obtained using backscattering detector. The hybrid particles were prepared when $[$ TEOS $]=0.54 \mathrm{M}$.
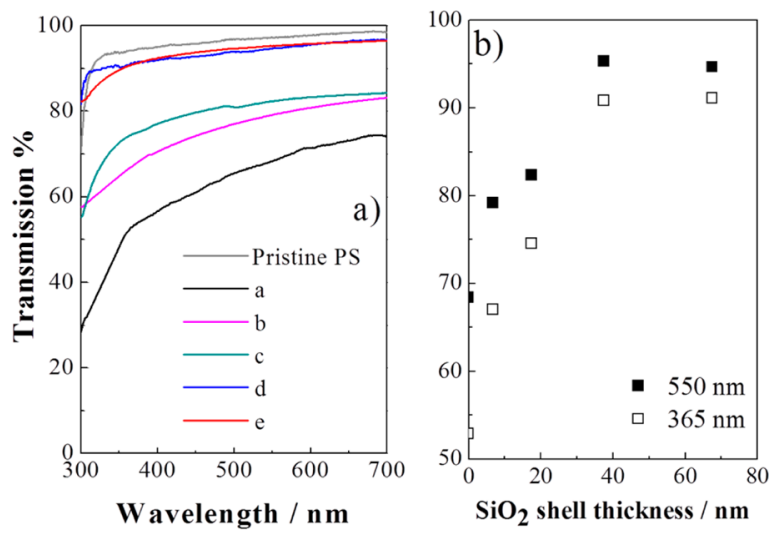

Figure 6. (a) UV-vis transmission spectra of the spin-coated freestanding PS composite films prepared by $\mathrm{CeO}_{2}$ and $\mathrm{CeO}_{2} @ \mathrm{SiO}_{2}$ nanoparticles at various shell thicknesses. Transmission spectra of nanocomposite films: (a) PS/neat $\mathrm{CeO}_{2}$ and (b-e) PS/ $\mathrm{CeO}_{2} @ \mathrm{SiO}_{2}$ with increasing shell thickness from bottom to top of (b) 6.5, (c) 17.5, (d) 37.5 , and (e) $67.5 \mathrm{~nm}$. The thickness of the composite films is nearly $100 \mu \mathrm{m}$. Transparency of pristine PS is shown a dashed line for the reason for comparison. The remarkable increase in transmission occurs at $37.5 \mathrm{~nm}$ shell thickness for $25 \mathrm{~nm} \mathrm{CeO}$ core. (b) Percent transmission of the all films as a function of $\mathrm{SiO}_{2}$ shell thickness at 550 and $365 \mathrm{~nm}$ wavelengths.

are obtained from freestanding films with a nearly $100 \mu \mathrm{m}$ thick films. Note that the thickness is similar to the thickness of A4 paper. $\mathrm{CeO}_{2}$ core content in all films was fixed at $3.0 \mathrm{wt} \%(0.6$ vol \%). The spectra are registered in the entire UV-visible region, and the percent transmission was recorded at two different wavelengths: one from the UV region at $365 \mathrm{~nm}$ and another from the visible region at $550 \mathrm{~nm}$, where the human eye has the highest sensitivity. The films are strongly absorbing in the UV region of the spectrum because of the presence of $\mathrm{CeO}_{2}$ and styrene groups in the PS. The former is a semiconductor whose band gap $(3.26 \mathrm{eV})$ falls into the energy of UV region, and the latter has a benzene ring that absorbs UV light. The transmission of the films at $365 \mathrm{~nm}$ shows a linear increase with shell thickness. At nearly $37.5 \mathrm{~nm}$, the transmission reaches the highest transmission among the composites. This result is compatible with the absorption spectra of the particles in THF where the percent transmission exhibits a remarkable increase, as shown in Figure 2. On the other hand, in the visible region, the films experience optical scattering and any intensity loss hints at the extent of optical scattering. Pristine PS film is transparent over the visible spectrum at about $96 \%$ transmission at normal incidence, and 
the remaining $4 \%$ can be attributed to surface reflection from air to film and scattering, most probably due to the density fluctuation remaining in the film structure in the course of film formation. The transmission of PS is shown with a dashed line for the sake of comparison. The incorporation of neat $\mathrm{CeO}_{2}$ causes a dramatic decrease of transmission to 53\%. A sharp increase in refractive index at the interface of individual neat particles or their large particle domains causes scattering. The usage of a ceria core-silica shell particle system increases the transmission even though the amount of ceria is identical in all samples. At $6.5 \mathrm{~nm}$ shell thickness, the transmission increases from 53 to $67 \mathrm{~nm}$, as shown in Table 1 . When the silica shell thickness was increased to $37 \mathrm{~nm}$, the transmission value reached $91 \%$, which is very close to the level of pristine PS. The thickness is almost identical to the one in the proposed theoretical calculation. This result can be considered as the validation of the index matching strategy for the preparation of transparent polymer/pigment particle composites.

As shown in Figure 6, the experimental scattering coefficient of composite films was evaluated at wavelengths of 532 and 365 $\mathrm{nm}$ from the experimental results as follows: $T=\exp (\beta s)$, where $T$ is transmission, $\beta$ is the extinction coefficient, and $s$ is the thickness of the films. $T$ and $s$ are measured, but $\beta$ can be calculated. The theoretical calculation of the coefficient of the hybrid particles is also studied for various silica shell thicknesses and wavelengths predicted by Effective Medium Theory. Figure $7 \mathrm{a}$ shows both the theoretical scattering and experimental
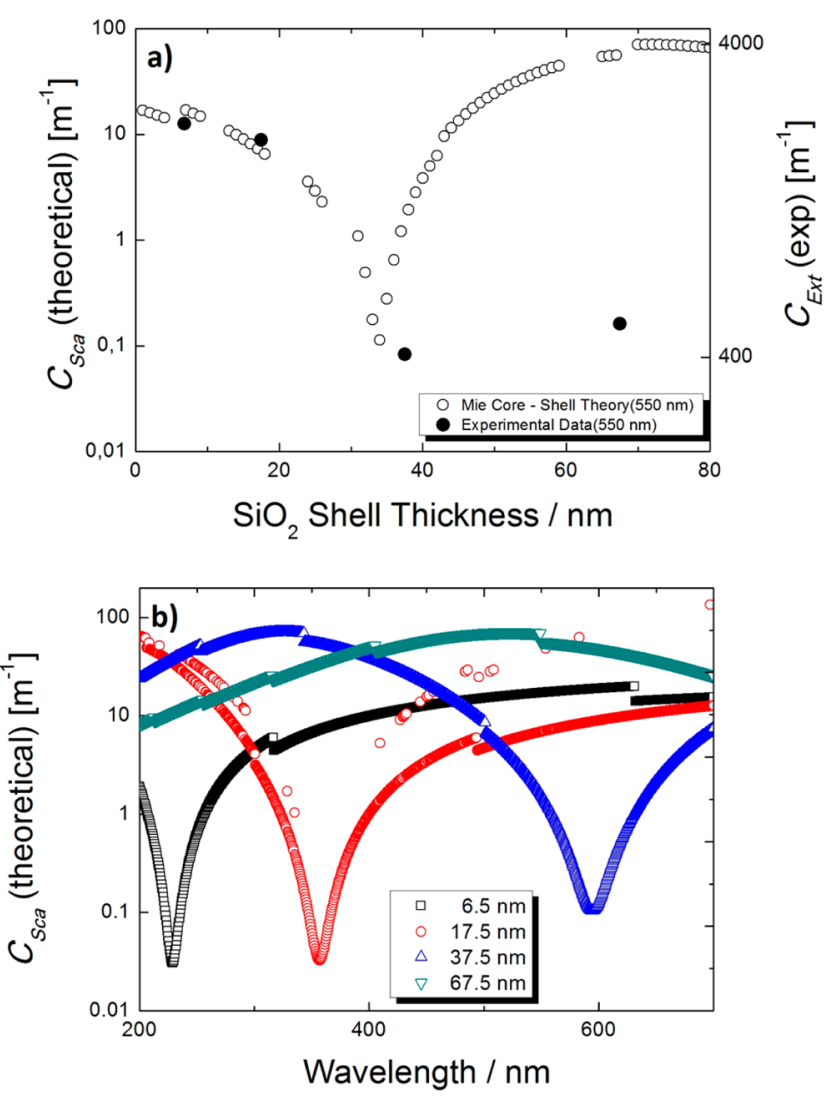

Figure 7. (a) Theoretical scattering (open points) and experimental (close points) extinction coefficient of $\mathrm{CeO}_{2} @ \mathrm{SiO}_{2}$ particles as a function of $\mathrm{SiO}_{2}$ shell thickness. Minimum point indicates the prediction of index-matching condition between core-shell particles and surrounding polymer matrix based on the effective medium theory. (b) Calculated coefficient of scattering at various wavelengths. extinction coefficients of $\mathrm{CeO}_{2} @ \mathrm{SiO}_{2}$ in PS as a function of shell thickness. In principal, a beam is attenuated both by scattering and absorption. Scattering is usually the dominant mode of extinction in such heterogeneous polymer/inorganic particle composite systems. For neat ceria particles, the scattering coefficient is high because the incident light experiences a sharp increase in the refractive index while propagating through the transparent matrix. Upon the integration of silica to the surface of the pigment core, the scattering coefficient decreases. This result suggests that the silica shell may reduce the extent of the refractive index mismatch between the core particles and the PS matrix. The scattering coefficient remarkably diminishes when the shell thickness is around $34 \mathrm{~nm}$. It should be noted that the lower the coefficient, the higher the transmission. This result is very close to the result we obtained with high transparency at 37.5 $\mathrm{nm}$ of shell thicknesses.

The quantitative comparison shows that the experimental values are nearly 2 orders of magnitude higher than the theoretical ones depending on the thickness of silica shell. There are three uncertainties that may cause this deviation. One major reason could be the presence of uncoated core particles. Although TEOS readily precipitates on the surface of ceria core, some amount of the core particles in the reaction mixture may be not coated and remain naked. These naked particles were rarely observed in electron microscopy and in aggregated state. These large aggregated particle domains consisting of individual core particles may cause strong optical scattering; thus scattering coefficient appears out higher. Another possible reason for this deviation could be the presence of pseudoaggregates of the $\mathrm{CeO}_{2}$ nanoparticles in the system. In the typical synthesis process, PVP-treated ceria particles are isolated from the dispersion medium via centrifugation. After drying step, the particles are redispersed into aqueous alkaline solution for silica coating. At this step, not all the particles are individually dispersed. There may be some particle domains still remaining in the aggregated state during silica coating such that the surface of these pseudoaggregates are covered rather than individual particles. Because the scattering intensity exponentially increases with the size of the scatterers (particle domains), scattering coefficient rapidly increases. The last reason could be the heterogeneity of the shell thickness over the ceria core particles. The theory assumes a uniform shell coating on the uniform size of the ceria core. However, from the experimental point of view, both the core diameter and the shell thickness exhibit size distribution as suggested by DLS measurements.

The experimental data were divided into 100 for the comparison with the theoretical ones in the same plot. The experimental and theoretical results exhibit similar trend particularly at short thickness. On the other hand, they show remarkable deviation from each other at a first glance at the thick silica shell. However, considering the reduction of experimental data by 100 , the deviation implies that both values become closer at this data point. In other words, the gap between the theoretical and experimental data points is reduced. The origin of this result could be the uniformity of silica coating at this shell thickness. Because high TEOS concentration is employed for the development of thick shell so that core particles may be evenly covered. Thus, the experimental extinction coefficient may approach the theoretical one. 
The thickness required for refractive index matching also varies with the wavelength of incident light. Figure $7 \mathrm{~b}$ presents the calculated scattering coefficient of the hybrid particles in PS at different wavelengths. When the shell thickness is $6.5 \mathrm{~nm}$ for a given $25 \mathrm{~nm}$ core diameter, the PS composite film has a minimum scattering coefficient at $220 \mathrm{~nm}$ and is accordingly transparent at this wavelength. With an increase of the shell thickness to $17.5 \mathrm{~nm}$, the minimum shifts to $360 \mathrm{~nm}$. At 37.5 $\mathrm{nm}$ shell thickness, the minimum appears at $600 \mathrm{~nm}$. A further increase in shell thickness to $67.5 \mathrm{~nm}$ does not give a minimum in extinction over the optical spectrum. Therefore, the scattering coefficient is remarkably reduced for a particular wavelength and for a given shell thickness.

Figure 8 shows photographic images of two PS composites: one prepared with neat $\mathrm{CeO}_{2}$ and another prepared with
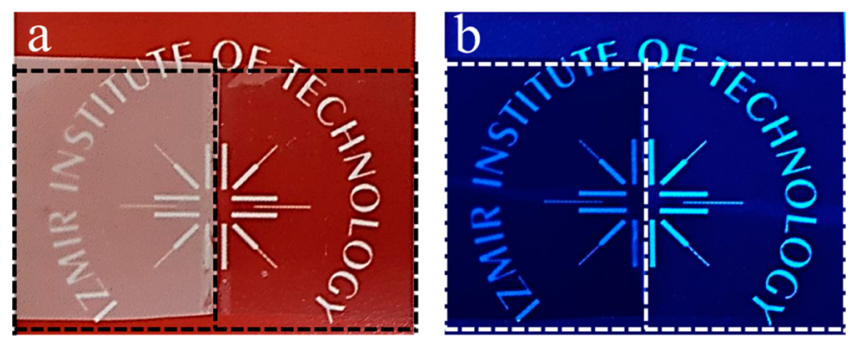

Figure 8. Photographic images of PS composites prepared with neat $\mathrm{CeO}_{2}$ and $\mathrm{CeO}_{2} @ \mathrm{SiO}_{2}$ particles under day- and UV-light $(\lambda=366$ $\mathrm{nm})$. The amount of $\mathrm{CeO}_{2}$ in both composites is $3.0 \%$ by mass. Dashed lines show the borders of the composite films for the guidance of eye. The thickness of the films is $\sim 100 \mu \mathrm{m}$.

$\mathrm{CeO}_{2} @ \mathrm{SiO}_{2}$ particles with $37.5 \mathrm{~nm}$ shell thickness under day(left) and UV-(right) light. The former composite is opaque under daylight and the latter is transparent, although they have identical $\mathrm{CeO}_{2}$ content. Under UV light, the former composite is absorbing, not surprising because semiconductors are absorbing UV light. On the other hand, the latter seems transparent under UV light. This observation validates the reduction of the extinction coefficient of the ceria core-silica shell hybrid particles over both UV-visible regions. The index matching strategy causes a dramatic improvement in optical clarity depending on the silica shell thickness and the wavelength of incident light over both the visible and UV regions.

\section{CONCLUSION}

Spherical and concentric ceria@silica core@shell hybrid particles with various shell thicknesses were fabricated using the Stöber method. The silica shell thickness was conveniently controlled within a broad range from 6.5 to $67.5 \mathrm{~nm}$ for different TEOS concentrations of $0.054-0.1080 \mathrm{M}$ in water. The interface between the ceria core particle and medium was found to play a key role in both the absorption and scattering features of the hybrid particles. The silica shell not only enhanced colloidal stability but also contributed to the transparency of the composite film. The thickness of the interface silica shell governs the scattering coefficient and molar absorptivity of the hybrid particles. The particles were nonabsorbing both in THF and PS under UV and nonscattering in PS over the visible region at $37.5 \mathrm{~nm}$ shell thickness for a fixed ceria core diameter at $25 \mathrm{~nm}$. The thickness required to achieve refractive index matching was calculated with the Effective Medium Theory. The result of the theoretical calculation is $34 \mathrm{~nm}$. Thus, the theory can be used to provide a reliable guideline for the design of null-extinction composites that is validated by the experimental results.

\section{ASSOCIATED CONTENT}

\section{S Supporting Information}

The Supporting Information is available free of charge on the ACS Publications website at DOI: 10.1021/acsami.5b09818.

MatLab code of calculation of extinction coefficient of the hybrid particles (PDF)

\section{AUTHOR INFORMATION}

\section{Corresponding Author}

*E-mail: mdemir@iyte.edu.tr. Tel.: +90 23275075 11. Fax: +902327507509.

\section{Present Address}

${ }^{\dagger}$ O.P. is currently at Biosensors and Bioelectronics Centre, IFM, Linköping University, 58183 Linköping, Sweden

\section{Author Contributions}

The manuscript was written through contributions of all coauthors. They have given approval to the final version of the manuscript.

\section{Funding}

M.M.D. acknowledges “Outstanding Young Investigator” grant of the Turkish Academy of Sciences (TÜBA-GEBIP 2013).

\section{Notes}

The authors declare no competing financial interest.

\section{ACKNOWLEDGMENTS}

The authors thank Center for Materials Research of Izmir Institute of Technology for microscopy work and E. Karabudak for his fruitful discussions.

\section{REFERENCES}

(1) Jin, X.; Goetz, M.; Wille, S.; Mishra, Y. K.; Adelung, R.; Zollfrank, C. A Novel Concept for Self-Reporting Materials: Stress Sensitive Photoluminescence in $\mathrm{ZnO}$ Tetrapod Filled Elastomers. Adv. Mater. 2013, 25 (9), 1342-1347.

(2) Khrenov, V.; Klapper, M.; Koch, M.; Mullen, K. Surface Functionalized $\mathrm{ZnO}$ Particles Designed for the Use in Transparent Nanocomposites. Macromol. Chem. Phys. 2005, 206 (1), 95-101.

(3) Demir, M. M.; Koynov, K.; Akbey, U.; Bubeck, C.; Park, I.; Lieberwirth, I.; Wegner, G. Optical Properties of Composites of PMMA and Surface-Modified Zincite Nanoparticles. Macromolecules 2007, 40 (4), 1089-1100.

(4) Dang, A.; Hui, C. M.; Matyjaszewski, K.; Bockstaller, M. R. Design and Fabrication Strategies for High Transparency Polymer Nanocomposites with Dynamic Tunable Optical Response. Proc. SPIE 2014, 9181.

(5) Lu, C. L.; Yang, B. High Refractive Index Organic-Inorganic Nanocomposites: Design, Synthesis and Application. J. Mater. Chem. 2009, 19 (19), 2884-2901.

(6) Bombalski, L.; Dong, H. C.; Listak, J.; Matyjaszewski, K.; Bockstaller, M. R. Null-Scattering Hybrid Particles Using Controlled Radical Polymerization. Adv. Mater. 2007, 19 (24), 4486-4490.

(7) Winey, K. I.; Vaia, R. A. Polymer Nanocomposites. MRS Bull. 2007, 32 (4), 314-319.

(8) Vaia, R. A.; Maguire, J. F. Polymer Nanocomposites with Prescribed Morphology: Going Beyond Nanoparticle-Filled Polymers. Chem. Mater. 2007, 19 (11), 2736-2751.

(9) Sanchez, C.; Julian, B.; Belleville, P.; Popall, M. Applications of Hybrid Organic-Inorganic Nanocomposites. J. Mater. Chem. 2005, 15 (35-36), 3559-3592. 
(10) Novak, B. M. Hybrid Nanocomposite Materials - Between Inorganic Glasses and Organic Polymers. Adv. Mater. 1993, 5 (6), $422-433$.

(11) Kickelbick, G. Concepts for the Incorporation of Inorganic Building Blocks into Organic Polymers on a Nanoscale. Prog. Polym. Sci. 2003, 28 (1), 83-114.

(12) Demir, M. M.; Wegner, G. Challenges in the Preparation of Optical Polymer Composites With Nanosized Pigment Particles: A Review on Recent Efforts. Macromol. Mater. Eng. 2012, 297 (9), 838863.

(13) Caseri, W. Inorganic Nanoparticles as Optically Effective Additives for Polymers. Chem. Eng. Commun. 2008, 196 (5), 549-572.

(14) Parlak, O.; Demir, M. M. Anomalous Transmittance of Polystyrene-Ceria Nanocomposites at High Particle Loadings. J. Mater. Chem. C 2013, 1 (2), 290-298.

(15) Anzlovar, A.; Orel, Z. C.; Zigon, M. Poly(methyl methacrylate) Composites Prepared by in Situ Polymerization Using Organophillic Nano-to-Submicrometer Zinc Oxide Particles. Eur. Polym. J. 2010, 46 (6), 1216-1224.

(16) Koziej, D.; Fischer, F.; Kranzlin, N.; Caseri, W. R.; Niederberger, M. Nonaqueous TiO2 Nanoparticle Synthesis: a Versatile Basis for the Fabrication of Self-Supporting, Transparent, and UV-Absorbing Composite Films. ACS Appl. Mater. Interfaces 2009, 1 (5), 1097-1104.

(17) Nussbaumer, R. J.; Caseri, W. R.; Smith, P.; Tervoort, T. Polymer-TiO2 Nanocomposites: A Route Towards Visually Transparent Broadband UV Filters and High Refractive Index Materials. Macromol. Mater. Eng. 2003, 288 (1), 44-49.

(18) Caseri, W. Nanocomposites of Polymers and Metals or Semiconductors: Historical Background and Optical Properties. Macromol. Rapid Commun. 2000, 21 (11), 705-722.

(19) Mackay, M. E.; Tuteja, A.; Duxbury, P. M.; Hawker, C. J.; Van Horn, B.; Guan, Z. B.; Chen, G. H.; Krishnan, R. S. General Strategies for Nanoparticle Dispersion. Science 2006, 311 (5768), 1740-1743.

(20) Kyprianidouleodidou, T.; Caseri, W.; Suter, U. W. Size Variation of Pbs Particles in High-Refractive-Index Nanocomposites. J. Phys. Chem. 1994, 98 (36), 8992-8997.

(21) Beecroft, L. L.; Ober, C. K. High Refractive Index Polymers for Optical Applications. J. Macromol. Sci., Part A: Pure Appl.Chem. 1997, A34 (4), 573-586.

(22) Chau, J. L. H.; Hsieh, C.-C.; Lin, Y.-M.; Li, A.-K. Preparation of Tansparent Silica-PMMA Nanocomposite Hard Coatings. Prog. Org. Coat. 2008, 62 (4), 436-439.

(23) Plat, M. J. Optical Efficiency Factors for Concentric Spheres. Appl. Opt. 1967, 6 (9), 1555-1558.

(24) Dang, A.; Ojha, S.; Hui, C. M.; Mahoney, C.; Matyjaszewski, K.; Bockstaller, M. R. High-Transparency Polymer Nanocomposites Enabled by Polymer-Graft Modification of Particle Fillers. Langmuir 2014, 30 (48), 14434-14442.

(25) Parlak, O.; Demir, M. M. Toward Transparent Nanocomposites Based on Polystyrene Matrix and PMMA-Grafted CeO2 Nanoparticles. ACS Appl. Mater. Interfaces 2011, 3 (11), 4306-4314.

(26) Tuzuner, S.; Demir, M. M. Dispersion of Organophilic Ag Nanoparticles in PS-PMMA Blends. Mater. Chem. Phys. 2015, 162, 692-699.

(27) Zou, H.; Wu, S. S.; Shen, J. Polymer/Silica Nanocomposites: Preparation, Characterization, Properties, and Applications. Chem. Rev. 2008, 108 (9), 3893-3957.

(28) Li, Y. Q.; Fu, S. Y.; Yang, Y.; Mai, Y. W. Facile Synthesis of Highly Transparent Polymer Nanocomposites by Introduction of Core-Shell Structured Nanoparticles. Chem. Mater. 2008, 20 (8), 2637-2643.

(29) Otanicar, T. P.; Phelan, P. E.; Taylor, R. A.; Tyagi, H. Spatially Varying Extinction Coefficient for Direct Absorption Solar Thermal Collector Optimization. J. Sol. Energy Eng. 2011, 133 (2), 024501.

(30) Tay, A. W. K. H. X. W. Real-Time Control of Photoresist Extinction Coefficient Uniformity in the Microlithography Process. Control Systems Technology, IEEE Transactions 2007, 15 (1), 99-105.

(31) Public domain software to be downloaded from http://imagej. net/Welcome.
(32) Wunderlich, W. Physical Constants of Poly(methyl meth-acrylate); J. Wiley \& Sons: New York, 1999; Vol. V/87.

(33) MATLAB, release 2013b; The MathWorks, Inc., Natick, MA.

(34) Graf, C.; Vossen, D. L. J.; Imhof, A.; van Blaaderen, A. A general method to coat colloidal particles with silica. Langmuir 2003, 19 (17), 6693-6700.

(35) Toki, M.; Chow, T. Y.; Ohnaka, T.; Samura, H.; Saegusa, T. Structure of Poly(vinylpyrrolidone)-Silica Hybrid. Polym. Bull. 1992, 29 (6), 653-660.

(36) Werner Stöber, A. F.; Bohn, E. Controlled growth of monodisperse silica spheres in the micron size range. J. Colloid Interface Sci. 1968, 26 (1), 62-69.

(37) Wang, M.-Q.; Yan, J.; Cui, H.-P.; Du, S.-G. Low Temperature Preparation and Characterization of $\mathrm{TiO} 2$ Nanoparticles Coated Glass Beads by Heterogeneous Nucleation Method. Mater. Charact. 2013, 76, 39-47.

(38) Liz-Marzan, L. M.; Giersig, M.; Mulvaney, P. Synthesis of Nanosized Gold-Silica Core-Shell Particles. Langmuir 1996, 12 (18), 4329-4335.

(39) Shah, K. W.; Sreethawong, T.; Liu, S. H.; Zhang, S. Y.; Tan, L. S.; Han, M. Y. Aqueous Route to Facile, Efficient and Functional Silica Coating of Metal Nanoparticles at Room Temperature. Nanoscale 2014, 6 (19), 11273-11281.

(40) Rogach, A. L.; Nagesha, D.; Ostrander, J. W.; Giersig, M.; Kotov, N. A. "Raisin bun"-Type Composite Spheres of Silica and Semiconductor Nanocrystals. Chem. Mater. 2000, 12 (9), 2676-2685.

(41) Cohren, C. F.; Huffman, D. R. Absorption and Scattering of Light by Small Particles; Wiley-VCH: Weinheim, Germany, 1998.

(42) Halliday, D.; Walker, J.; Resnick, R. Fundamentals of Physics; Wiley: New York, 1970.

(43) Dixon, K. W., In Polymer Handbook, 4th ed.; Brandgup, J., Immergut, E. H., Grulke, E. A., Eds.; J. Wiley \& Sons: New York, 1999. 